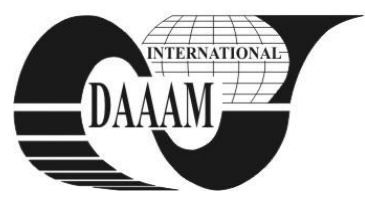

\title{
BIFURCATION ANALYSIS OF DIRECT-CURRENT MICRODRIVE
}

\author{
VONDRUS, J[iri] \& CIPIN, R[adoslav]
}

\begin{abstract}
This study deals with bifurcation analysis of directcurrent microdrives. It is focused on monitoring the stability of the supply current of the DC motor depending on the input parameters (regulator settings). The measurement is based on a mathematical model of a DC motor which is powered by the PI controller
\end{abstract}

Key words: bifurcation, DC motor, mathematical simulation, PI controller, PWM

\section{INTRODUCTION}

This paper deals with the kind of bifurcation we can find in electro-mechanics; to be specific, it deals with the analysis of bifurcation diagrams in a system of a DC motor with a PI regulator.

Bifurcation images belong to a set of fractals. Fractals are images similar to each other, the shape of which seems complicated at first glance, but in fact they are generated by a repeating row of images created with simple rules. Bifurcation is a name for such change of an output parameter of the system which results in separation of the original trajectory of the parameter development to several new structures of various levels - owing to even a slight change of the input parameters.

Bifurcation analysis can be used at both electrical and nonelectrical quantities (tension, current, speed, torque, etc.) depending upon the change of one or more of the parameters (supply voltage, temperature, friction coefficient, frequency, etc.). Based on the results can be customized control or predict failure conditions (diagnosis).

\section{BIFURCATION}

Bifurcation is associated with natural phenomena (splitting water flows), medicine (vascular and neurological networks), mathematics, etc. The available information shows that the bifurcation is associated with the area of electrical, mechanical or electromechanical fields at least. The bifurcation or the bifurcation analysis can be applied to any natural or scientific study. Whatever the nonlinear dynamic system, which appears in certain period of time as a stable - deterministic, in the long term behaves unpredictably (chaotically), and it is sensitive to the change of initial conditions.

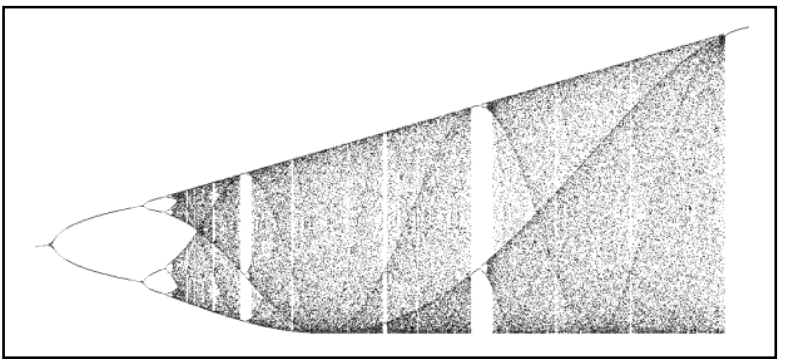

Fig. 1. General bifurcation diagram
The process of a bifurcation diagram can be exemplified, as seen in Fig. 1. Detailed scrutiny will reveal areas where trajectory separation occurs - the so-called critical points. The bifurcation diagram also shows evident regularities restricting limits and "white windows", where under certain conditions and "order" in the otherwise chaotic design can occur.

Practical measurement can turn out problematic when searching for bifurcation points; the swing of the observed quantity may sometimes be so little that it vanished because of the small accuracy of the meter. In general, it is possible to find 2 or 3 bifurcation points during a practical measurement with the trajectory splitting up to 4 or 8 levels.

The trajectory split area is not parametrically infinitely short. Gradual trajectory splits can be used for a hopeful prediction of the system's state.

\section{COMPUTER SIMULATION}

The easiest way to carry on a system diagnosis on a PC and seek out bifurcation points can be done by the help of a mathematical model of a DC motor with a PI speed regulator with current limiter. This DC model stems from the voltage equation for a DC engine with separate excitation (a permanent magnet, for instance).

$$
\begin{aligned}
& u_{a}=R_{a} \cdot i_{a}+L_{a} \cdot \frac{d i_{a}}{d t}+u_{i} \\
& c \cdot \emptyset \cdot i_{a}=J \cdot \frac{d \omega}{d t} \cdot T_{r}+B \cdot \omega
\end{aligned}
$$

where:

$$
\begin{array}{llll}
u_{a} & \text { supply voltage }[\mathrm{V}] & c \cdot \emptyset & \text { speed constant }[\mathrm{V} \cdot \mathrm{s}] \\
i_{a} & \text { current [A] } & \omega & \text { speed }\left[\mathrm{s}^{-1}\right] \\
R_{a} & \text { winding resistance }[\Omega] & T_{r} & \text { reaction torque }[\mathrm{N} \cdot \mathrm{m}] \\
L_{a} & \text { winding inductance }[\mathrm{H}] & B & \text { friction coefficient }[\mathrm{N} \cdot \mathrm{m} \cdot \mathrm{s}] \\
t & \text { time }[\mathrm{s}] & J & \text { rotor inertia }\left[\mathrm{kg} \cdot \mathrm{m}^{2}\right]
\end{array}
$$

A computer model works with real values that were measured on a DC motor Dynamo Sliven PIVT 6-25/3A at reduced supply voltage to $14 \mathrm{~V}$, nominal voltage is $30 \mathrm{~V}$. All measured parameters are listed below:

$\begin{array}{ll}\text { Nominal supply voltage } & U_{N}=30 \mathrm{~V} \\ \text { Nominal current } & I_{N}=3 \mathrm{~A} \\ \text { Reduced supply voltage } & U_{R}=14 \mathrm{~V} \\ \text { Reduced current } & I_{R}=0,82 \mathrm{~A} \\ \text { Winding resistance } & R_{A}=2,245 \Omega \\ \text { Winding inductance } & L_{A}=4,6734 \mathrm{mH} \\ \text { Speed constant } & \mathrm{c} \cdot \emptyset=0,068977 \mathrm{~V} \cdot \mathrm{s} \\ \text { Nominal speed } & n_{N}=3000 \mathrm{~min}^{-1} \\ \text { Reduced speed } & n_{R}=1685 \mathrm{~min}^{-1} \\ \text { Friction coefficient } & B=0,028281 \mathrm{~N} \cdot \mathrm{m} \cdot \mathrm{s} \\ \text { Rotor inertia } & J=5,964 \cdot 10^{-5} \mathrm{~kg} \cdot \mathrm{m}^{2}\end{array}$




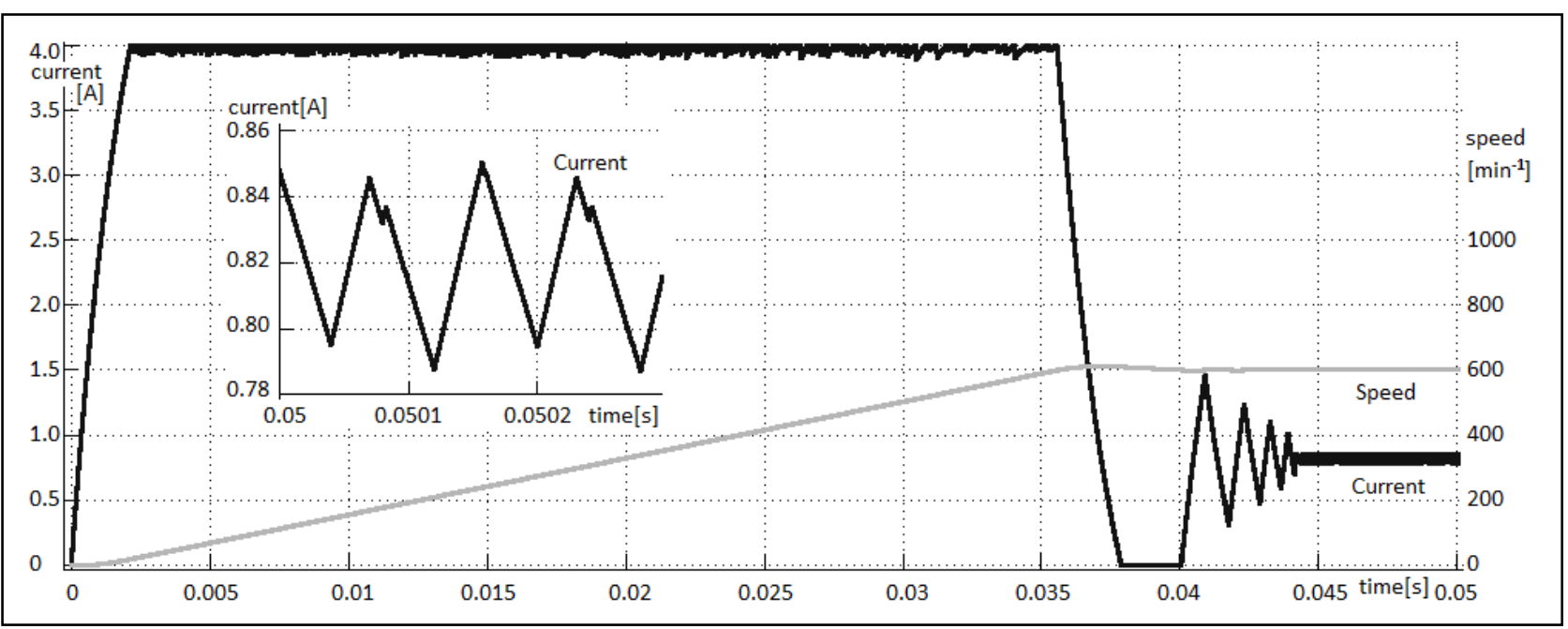

Fig. 2. Simulation results

\section{SIMULATION RESULTS}

The model is designed to allow change in any parameters in selected range with the specified step. It allows a simulation of the effect of setting the controller (proportional or integral gain, PWM frequency, supply voltage, etc.) to the observed values, but also the influence of changes of motor parameters (windings resistance or inductance, rotor inertia, friction coefficient, etc.).

Fig. 2. shows the current and speed in the simulation, the desired speed value was set to $600 \mathrm{~min}^{-1}$ with the current limiting 4A, regulator frequency was set to $25 \mathrm{kHz}$. The detailed view shows that the current waveform is curled, it is caused by setting the controller and the individual values (parameters) of the system. Engine speed is also curled, but the value of ripples is very small (hundredths of a turn).

Bifurcation diagram showing the stability or instability of the system depending on the parameter can be obtained after a series of simulations during which all parameters remaining constant (there is only change of the examined parameter in the desired range). In the bifurcation diagram is altered parameter shown at $\mathrm{X}$-axis, the output values of the monitored dynamic systems are plotted to $\mathrm{Y}$-axis.

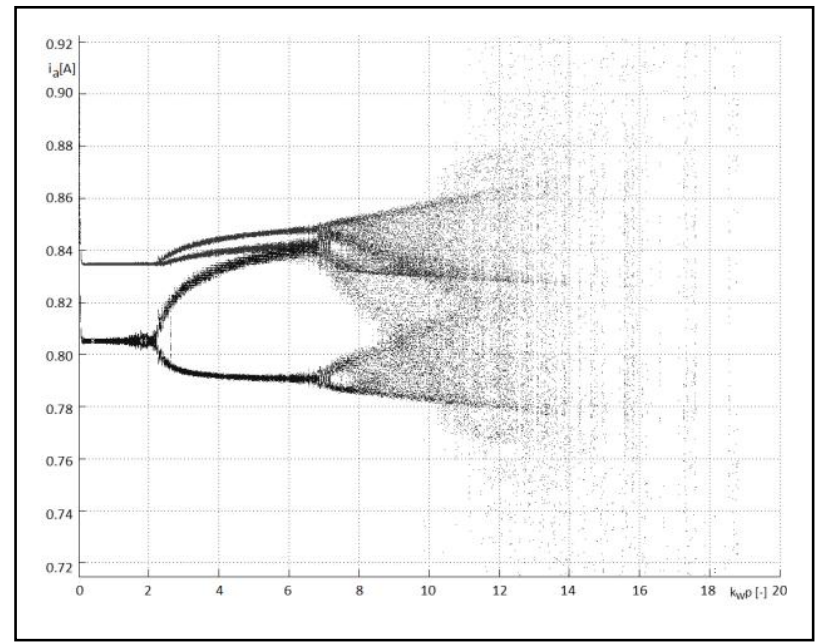

Fig. 3. Bifurcation diagram of the supply current $I_{a}$

The example of bifurcation diagram of the supply current (altered parameter is proportional gain of speed controller) is shown at Fig. 3. Detailed scrutiny will reveal areas where trajectory separation occurs and ripples amplitude is increasing with the values of altered parameter.

\section{CONCLUSION}

Bifurcation analysis can be used - among others - for designing and setting up the regulator parameters. The initial values can be determined for example by applying the optimization method. Using a row of successive simulations for the sweep of individual parameters will give us a series of bifurcation diagrams that allows us to re-set the regulator in order for the working point to be kept in the stable area as far as the current and the speed is concerned, while maintaining the demanded output values of the engine speed.

Similarly, it is possible to carry on the simulation of the regulator's behavior while swinging other parameters, such as the amplification of the current or speed probe, the fluctuation of the regulator's frequency, or the supply voltage ripple.

It is of course possible to observe bifurcation on real systems as well, not only on computer simulation. Problem with real measuring, though, usually lies in the sensitivity of used devices, because the swing between the levels may be smaller than what the device is capable of determining.

Bifurcation analysis of an electromechanical system can be considered very hopeful when observing the changes of the parameters of a non-linear dynamic system, especially from the viewpoint of the predictability of failure states.

\section{ACKNOWLEDGEMENTS}

This work has been created at the support of the projects MSM 0021630518 and OP VK CZ.1.07/2.3.00/09.0162.

\section{REFERENCES}

Basak, B., Parui, S., (2009), Bifurcation and chaos in current mode controlled DC drives in continuous and discontinuous conduction mode of operation, In TENCON 2009, Singapore, pp. 1 - 6, ISBN: 978-1-4244-4546-2

Darrigol, O., (2005), The Genesis of the theory of relativity, Séminaire Poincaré 1, Paris: Rehseis

Chau, K. T.; Chen, J. H.; Chan, C. C., (1997), Dynamic bifurcation in DC drives, In Power Electronic Specialists Conference, PESC '97 Record, $28^{\text {th }}$ Annual IEEE, St. Louis, ISBN:0-7803-3840-5, pp. 1330 - 1336, St. Louis

Kratochvil, C., and col, (2008), Bifurcation and chaos in engineering systems and their modeling, Brno UT AVCR in Prague, 978-80-214-3720-3, Prague

Kuroe, Y., Hayashi, S., (1989), Analysis of bifurcation in power electronic induction motor drive systems, In Power Electronics Specialists Conference, PESC '89 Record., 20th Annual IEEE, Milwaukee, WI, pp. 923 - 930 